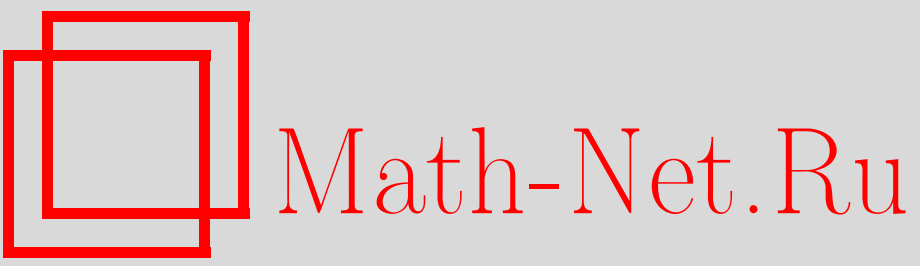

Э. Л. Аэро, Слабозатухающие медленные моды ориентационных нелинейных колебаний в жидких кристаллах в дестабилизирующих структуру магнитных полях, ТМФ, 1997, том 111, номер 1, 132-143

DOI: https://doi.org/10.4213/tmf995

Использование Общероссийского математического портала Math-Net.Ru подразумевает, что вы прочитали и согласны с пользовательским соглашением

http://www.mathnet.ru/rus/agreement

Параметры загрузки:

IP: 54.237 .59 .107

26 апреля 2023 г., $14: 26: 37$ 
ТЕОРЕТИЧЕСКАЯ

И МАТЕМАТИЧЕСКАЯ

ФИЗИКА

Том 111, № 1

апрель, 1997

Э. Л. Аэро*

\section{СЛАБОЗАТУХАЮШИЕ МЕДЛЕННЫЕ МОДЫ ОРИЕНТАЦИОННЫХ НЕЛИНЕЙНЫХ КОЛЕБАНИЙ В ЖИДКИХ КРИСТАЛЛАХ В ДЕСТАБИЛИЗИРУЮШИХ СТРУКТУРУ МАГНИТНЫХ ПОЛЯХ}

Нелинейные инерционные колебания кручения структуры нематического жидкого кристалла в дестабилизируюшем магнитном поле имеют низкочастотные моды, обладающие аномально низким затуханием. Соответствующее решение уравнения типа синус-Гордон с учетом вязкого вращательного трения описывает переходы через потенциальный барьер между двумя состояниями равновесия. При колебаниях с амплитудами вблизи уровня, необходимого для преодоления барьера, время затухания, отнесенное к периоду колебаний, логариффмически расходится.

\section{ВВЕДЕНИЕ}

В теории нелинейных колебаний нематических жидких кристаллов (нематиков) пренебрегают собственно ориентационными модами, связанными с врашательной инерцией молекул или их кластеров $[1,2]$. При этом с полным основанием ссылаются на ее малую величину и значительное внутреннее врашательное трение. Этим объясняется достаточно высокий порог возникновения распространяющихся волн, превышающий частоты порядка $10^{8}$ Гц. Однако успехи нелинейной теории сосредоточенных волн, достигнутые в других областях физики, дают повод к пересмотру “линейной идеологии" и в нематодинамике. Локализованный характер солитоноподобной волны в случае высокой скорости ее распространения позволяет ожидать малых потерь энергии, отнесенных ко всей области и периоду колебания. Особый интерес представляют и медленные низкочастотные моды, у которых этот период $(2 T)$ достаточно велик.

Поиск подобных мод требует точного анализа нелинейных уравнений нематодинамики. В данной работе это будет сделано вначале для чисто упругого случая с последующим приближенным рассмотрением эффектов диссипации. На примере связанных волн будут рассмотрены колебания в ограниченной области - плоскопараллельном слое с жесткими границами. Их можно было бы назвать стоячими волнами, однако в нелинейном случае они не сводятся к суперпозишии двух волн, бегуших навстречу друг другу.

${ }^{*}$ Институт проблем машиноведения РАН 
Ограничимся рассмотрением лишш деформаций кручения, полагая, что поле директора $\mathbf{l}(x, t)$ подчиняется условию $\mathbf{l} \operatorname{rot} \mathbf{l}=0$. Примем также, что структура нематика не имеет предварительной закрутки.

\section{1. ОСНОВНЫЕ УРАВНЕНИЯ}

Интересующие нас моды возникают, в частности, в дестабилизирующем магнитном поле (напряженности $H$ ), которое направлено ортогонально граничной ориентации директора $\mathbf{l}_{s}$, одинаковой для обеих границ $x= \pm(h / 2)$. Векторы $\mathbf{H}$ и $\mathbf{l}$ лежат в плоскости слоя. Они изображены на рис. 1 , где также показано, как направлено стабилизируюшее поле $\mathbf{H}_{\|}$, не деформируюшее структуру нематика.

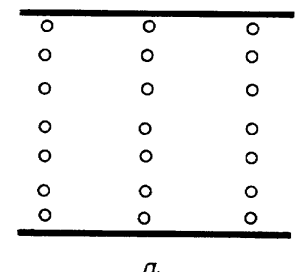

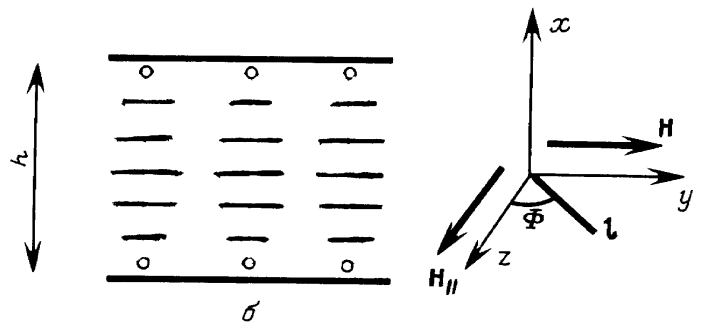

Рис. 1. Недеформированная (а) и деформированная (б) структуры слоя нематика.

Кружочки на рис. 1а обозначают “торцы” молекул, а горизонтальные линии на рис. $1 б$ - проекции молекул на плоскость рисунка. Имеем

$$
H_{z}=H, \quad H_{x}=H_{y}=l_{y}=l_{x}=0, \quad l_{z}=\cos \Phi, \quad(\mathbf{H l})=H \sin \Phi .
$$

Отсюда видно, что $\Phi(x, t)$ - это угол взаимной ориентации векторов $\mathbf{H}$ и $\mathbf{1}$, лежаших в плоскости слоя $y z$. Он является дополнительным к углу $\theta$ между этими векторами $(\theta+\Phi=\pi / 2)$.

На границах слоя будем считать, что

$$
\Phi\left( \pm \frac{h}{2}, t\right)=0
$$

В статике подобное расположение полей $\mathbf{l}$ и $\mathbf{H}$ приводит к переходу Фредерикса, т.е. к потере устойчивости первоначально однородной конфигурации поля $\mathbf{l}=0$ после достижения порогового значения величины $H h=\mu_{c}=\frac{\pi}{2} \sqrt{\frac{k}{\chi}}$. В результате возникает закрученное состояние структуры нематика.

Будем интересоваться большими колебаниями углового поля $\Phi(x, t)$ как вблизи, так и вдали от порога Фредерикса $\mu_{c}$ как с той, так и с другой стороны от него. При этом будут рассмотрены симметричные (во времени) колебания.

Общее уравнение крутильных колебаний имеет вид [3]

$$
J \frac{\partial^{2} \Phi}{\partial t^{2}}=k \frac{\partial^{2} \Phi}{\partial x^{2}}+\frac{1}{2} \chi H^{2} \sin 2 \Phi-\gamma \frac{\partial \Phi}{\partial t},
$$


здесь $J$ - средний (по малому объему) молекулярный или кластерный момент инерции, $k$ - упругий модуль кручения Озеена-Франка, $\gamma$ - врашательная вязкость, $\chi$ - положительная диамагнитная анизотропия.

Выбор кручения (в отличие от изгибов) позволяет избежать гидродинамических $(\sim \operatorname{rot} \mathbf{v})$ диссипативных слагаемых в $(1.3)$. Влияние диссипативного члена $(\sim \gamma)$ будет выявлено в последнем разделе статьи. Без учета этого члена имеем вместо (1.3) уравнение упругих инерционных колебаний

$$
J \frac{\partial^{2} \Phi}{\partial t^{2}}=k \frac{\partial^{2} \Phi}{\partial x^{2}}+\frac{1}{2} \chi H^{2} \sin 2 \Phi
$$

Оно отличается от широко известного в различных областях физики уравнения синусГордон лишь знаком перед последним слагаемым. Хотя это не сказывается на технике построения решения, его вид и физический смысл оказываются иными. Заметим, что переход к противоположному знаку перед этим слагаемым получается в случае, если вместо дестабилизируюшего рассматривается магнитное поле $\mathbf{H} \| \mathbf{1}_{s}$, стабилизирующее однородную начальную структуру. При этом переходов Фредерикса, конечно, не возникает.

\section{2. СИММЕТРИЧНЫЕ КОЛЕБАНИЯ}

В отличие от статики динамические деформации поля $\mathbf{l}(x, t)$ возможны как в докритической области $\left(h H<\mu_{c}\right)$, так и за ее пределами $\left(h H>\mu_{c}\right)$. В данном разделе рассмотрим симметричные периодические колебания, которые совершаются около состояния статического равновесия $\Phi=0$ и характеризуются амплитудой $\Phi_{e}$ в центре слоя $(x=0)$. В моменты времени $t=0, t=T / 2, t=T$ имеем, таким образом, соотношения

$$
\begin{aligned}
& \Phi_{e}=\Phi(0,0)=\Phi(0, T), \\
& \Phi(x, T / 2)=0,
\end{aligned}
$$

здесь $T$ - полупериод колебаний.

Построение решения уравнения (1.4), удовлетворяющего условиям (1.2), (2.1), (2.2), будет основано на своеобразном методе разделения переменных, так же как для уравнения синус-Гордон. Согласно [4] нужно положить вначале

$$
\operatorname{tg} \frac{\Phi(x, t)}{2}=\Phi_{1} / \Phi_{2}, \quad \Phi_{1}=\Phi(t), \quad \Phi_{2}=\Phi_{2}(x)
$$

После подстановки (2.3) в (1.4) получим для функций $\Phi_{1}$ и $\Phi_{2}$ два обыкновенных дифференциальных уравнения

$$
\begin{gathered}
\left(\partial \Phi_{1} / \partial t\right)^{2}=a^{2}\left(A_{1}-\Phi_{1}^{2}\right)\left(B_{1}+\Phi_{1}^{2}\right) \\
\left(\partial \Phi_{2} / \partial x\right)^{2}=a^{2}\left(A_{2}+\Phi_{2}^{2}\right)\left(\Phi_{2}^{2}-B_{2}\right)
\end{gathered}
$$

Они имеют решения в эллиптических функциях. 
В оригинальной работе [4] в этих уравнениях справа в двух полиномах четвертой степени содержатся три независимые константы. Здесь, так же как это было сделано нами уже ранее [5], введено вместо трех независимых $(a, b, c)$ пять взаимосвязанных констант $a^{2}, A_{1}, A_{2}, B_{1}, B_{2}$ при сохранении степени полиномов. Выбирая различным образом знаки в круглых скобках, можно расширить круг эллиптических функций, из которых строится решение. В частности, знаки в (2.4) выбраны применительно к задаче о симметричных колебаниях. В результате вторичной подстановки в основное уравнение (1.4) имеем

$$
A_{1} B_{1}=A_{2} B_{2}, \quad a^{2}\left(A_{1}-B_{1}\right)-a^{2}\left(A_{2}-B_{2}\right)=1 .
$$

При этом возникают два дисперсионных соотношения (2.5), которые будут раскрыты далее. Отметим, что в [5] мы фактически обобшили прием, предложенный ранее в работе [6], где получено не два, а одно дисперсионное соотношение. Можно показать, что дальнейшее обобшение в этом смысле невозможно.

Вид уравнений (2.4) в точности соответствует нормальной форме эллиптических интегралов, которые табулированы [7]. Пользуясь ею, выпишем в явном виде конкретные функции $\Phi_{1}$ и $\Phi_{2}$, с помошью которых получается решение (2.3), удовлетворяюшее граничным условиям (1.2),

$$
\Phi_{1} \sim \mathrm{cn}\left[\frac{t}{T} 2 m K\left(\nu_{1}\right)\right], \quad \Phi_{2} \sim \mathrm{cn}\left[\frac{x}{h} 2 n K\left(\nu_{2}\right)\right], \quad n, m=1,2,3, \ldots
$$

При удовлетворении граничным условиям используется свойство эллиптического косинуса $\operatorname{cn}[2 n K(\nu)]=0, n=1,2,3, \ldots$ Для полного эллиптического интеграла 1-го рода $K(\nu)$, играющего здесь роль четверти периода, находим

$$
K(0)=\pi / 2, \quad K(1) \rightarrow \infty, \quad 0 \leqslant \nu \leqslant 1 .
$$

Ясно, что имеет место дискретный пространственно-временной спектр колебаний. В силу его простоты целесообразно будет далее рассмотреть основную моду $(m, n=1)$ и основные периоды $2 h, 2 T$. Более короткие периоды характерны для тех частных решений, которые возникают при энергетически более высоких уровнях возбуждения колебаний. Их анализ аналогичен излагаемому ниже.

Заметим еше, что построенное решение уравнения (1.4) не единственное. В частности, возможно найти решение и для несимметричных (по времени) колебаний.

Итак, решение для симметричных колебаний (основной моды стоячих волн) в докритической области $\left(h H<\mu_{c}=\frac{\pi}{2} \sqrt{k / \chi}\right)$ имеет вид

$$
\operatorname{tg} \frac{\Phi(x, t)}{2}=\left(\nu_{1} \nu_{2}\right)^{1 / 2}\left[\left(1-\nu_{1}^{2}\right)\left(1-\nu_{2}^{2}\right)\right]^{-1 / 4} \operatorname{cn}\left(\frac{2 t}{T} K_{1}\right) \operatorname{cn}\left(\frac{2 x}{h} K_{2}\right)
$$

где

$$
K_{1}=K\left(\nu_{1}\right), \quad K_{2}=K\left(\nu_{2}\right), \quad \nu_{1}^{2}=A_{1} /\left(A_{1}+B_{1}\right), \quad \nu_{2}^{2}=A_{2} /\left(A_{2}+B_{2}\right) .
$$


Здесь, кроме констант, которые уже конкретизированы на основании граничных условий, имеются еще две: $\nu_{1}$ и $\nu_{2}$. Это модули эллиптических интегралов $K\left(\nu_{1}\right)$ и $K\left(\nu_{2}\right)$. Легко видеть, что, с одной стороны, $\nu_{1}$ и $\nu_{2}$ характеризуют степень нелинейности задачи - при $\nu_{1,2} \rightarrow 0$ угол $\Phi$ также становится малым, эллиптические косинусы переходят в круговые и выражение (2.7) переходит в гармоническое решение линейного уравнения. $\mathrm{C}$ другой стороны, $\nu_{1}$ и $\nu_{2}$ связаны с амплитудой нелинейных колебаний $\Phi_{e}-$ наибольшим угловым отклонением в центре слоя, которое по условию достигается в момент времени $t=0$. При этом оба косинуса в (2.7) обращаются в единицу, и это выражение дает

$$
\operatorname{tg} \frac{\Phi_{e}}{2}=a=\left(\nu_{1} \nu_{2}\right)^{1 / 2}\left[\left(1-\nu_{1}^{2}\right)\left(1-\nu_{2}^{2}\right)\right]^{-1 / 4}, \quad \Phi_{e}=\Phi(0,0),
$$

здесь $a$ - тангенс-амплитуда. Через четверть периода $(t=T / 2)$ все выражение $(2.7)$ в соответствии с условием сn $K=0$ обращается в нуль, и поэтому система проходит через состояние равновесия $\Phi=0$. Это дополнительное условие (по отношению к начальному условию $\left.\Phi_{e}=\Phi(0,0)\right)$ необходимо и выделяет решение $(2.7)$ из всего набора частных решений.

Обратимся к анализу дисперсионных соотношений (2.5). Первое из них определяет фазовую скорость как функцию параметров формы колебаний $\nu_{1}$ и $\nu_{2}$, именно

$$
\frac{C^{2}}{C_{0}^{2}}=\frac{\nu_{2} \sqrt{1-\nu_{2}^{2}}}{\nu_{1} \sqrt{1-\nu_{1}^{2}}} \frac{K_{2}^{2}}{K_{1}^{2}}, \quad C=\frac{h}{T}, \quad C_{0}=\sqrt{\frac{k}{J}},
$$

где $C$ - фазовая скорость, $C_{0}$ - ее значение в нулевом магнитном поле.

Второе соотношение в (2.5) раскрывается в виде

$$
\frac{\left(1-2 \nu_{2}^{2}\right) K_{2}^{2}}{h^{2} / h_{\chi}^{2}}-\frac{\left(1-2 \nu_{1}^{2}\right) K_{1}^{2}}{T^{2} / T_{\chi}^{2}}=1, \quad h_{\chi}^{2}=\frac{k}{\chi H^{2}}, \quad T_{\chi}^{2}=\frac{J}{\chi H^{2}},
$$

здесь $h_{\chi}, T_{\chi}$ - характерные пространственно-временные масштабы.

Соотношениями $(2.10),(2.11)$ устанавливается связь пространственно-временных масштабов колебаний $h, T$ с параметрами $\nu_{1}$ и $\nu_{2}$. Это линейная система относительно величин $h^{-2}$ и $T^{-2}$. Решая ее, получим

$$
\begin{aligned}
& \frac{h^{2}}{h_{\chi}^{2}}=\left[1-2 \nu_{2}^{2}+\left(2 \nu_{1}^{2}-1\right) \frac{\nu_{2} \sqrt{1-\nu_{2}^{2}}}{\nu_{1} \sqrt{1-\nu_{1}^{2}}}\right] K_{2}^{2}, \\
& \frac{T^{2}}{T_{\chi}^{2}}=\left[2 \nu_{1}^{2}-1+\left(1-2 \nu_{2}^{2}\right) \frac{\nu_{1} \sqrt{1-\nu_{1}^{2}}}{\nu_{2} \sqrt{1-\nu_{2}^{2}}}\right] K_{1}^{2}, \quad \nu_{1}>\nu_{2} .
\end{aligned}
$$

Неравенство в (2.13) связано с необходимой положительностью выражений в квадратных скобках справа в обоих соотношениях. Используя наряду с ними выражение (2.9) для амплитуды $a$, можно в принципе исключить из этой системы трех уравнений два параметра $\nu_{1}$ и $\nu_{2}$. Тогда получим одно уравнение для семейства ветвей колебаний вида $\omega=\omega\left(m^{-1}, a\right)$, где $a$ играет роль параметра семейства, а $\omega$ и $m^{-1}$ - безразмерные частота и волновое число:

$$
\omega=T_{\chi} / T, \quad m^{-1}=h_{\chi} / h=\mu_{\chi} / h H, \quad \mu_{\chi}=\sqrt{k / \chi} .
$$


Заметим, что характерные масштабы $T_{\chi}, h_{\chi}$ зависят согласно $(2.11)$ не только от свойств среды, но и от напряженности магнитного поля. Обратное волновое число $m$ можно рассматривать и как граничный магнитный потенциал $h H$, отнесенный к характерному $-\mu_{\chi}$. Статическому порогу устойчивости однородной ориентации $\Phi=0$ соответствует значение $m=\pi / 2$ или $h H=(\pi / 2) \mu_{\chi}$. В аналитическом виде функцию $\omega\left(m^{-1}, a\right)$ выразить не удается. По результатам машинного счета на рис. 2 представлены ветви колебаний, каждая из которых соответствует заданному значению тангенс-амплитуды $a=\operatorname{tg}\left(\Phi_{e} / 2\right)$. Их обсуждение будет дано в следующем разделе.

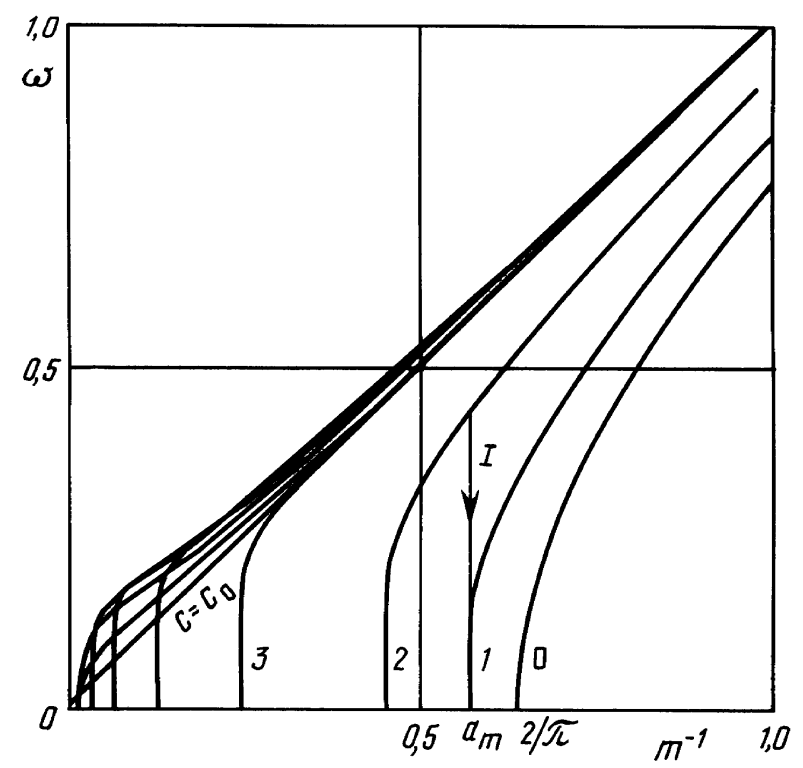

Рис. 2. Дисперсионные ветви основной моды симметричных колебаний в дестабилизирующем поле $H$.

\section{3. ОСОБЕННОСТИ НИЗКОЧАСТОТНОЙ ДИСПЕРСИИ}

Ветви колебаний в дестабилизируюшем поле при различных значениях амплитуды $a$ представлены на рис. 2. Некоторые из них напоминают гиперболу. Каждая начинается в некоторой точке на оси абсцисс $m_{a}^{-1}$ (при $\omega=0$ ) и стремится к асимптоте $\omega=C_{0} m^{-1}$, единой для всех ветвей. Она также является ветвью колебаний только в нулевом магнитном поле и исходит из начала координат. Наиболее удаленная от начала координат ветвь начинается в точке $m_{0}^{-1}=2 / \pi$. Она соответствует предельно мальм колебаниям. Все остальные ветви $(1,2,3)$ лежат на рис. 2 выше и левее. Им отвечают следующие значения угловой амплитуды $\Phi_{e}$ и тангенс-амплитуды $a: \Phi_{e}=26^{\circ}, a=0.5$ (ветвь 1 ); $\Phi_{e}=45^{\circ}, a=1$ (ветвь 2$) ; \Phi_{e}=67.5^{\circ}, a=2.4$ (ветвь 3 ).

Точка $m_{a}^{-1}$ делит ось абсцисс на две области. Область справа соответствует равновесным состояниям структуры. Слева же вплоть до начала координат располагаются точки бифуркаций, которые отвечают пределам статической устойчивости структуры. Рассмотрим эти состояния, реализуюшиеся при $\omega=0$, подробнее. 
Каждая ветвь, исходящая из точки этой области, соответствует колебаниям определенной амплитуды $a$. Поскольку они уходят в сторону больших значений $m^{-1}$ (малых $\left.m=h H / \mu_{\chi}\right)$, то колебания данной амплитуды $a$ сушествуют, когда абсциссы точек ветвей удовлетворяют неравенству

$$
m^{-1} \geqslant m_{a}^{-1} \text { или } m \leqslant m_{a}, \quad \omega=0 .
$$

Величина $m_{a}^{-1}$ является порогом, нижним пределом возбуждения колебаний данной амплитуды. Зависимость этого порога от амплитуды следует из анализа соотношений $(2.12),(2.13)$ с учетом (2.9). Положив в $(2.13) \omega=0$, получим $\nu_{1}=1$. Обрашаясь к (2.9), находим, что если $a$ - ограниченная величина, то $\nu_{2}=0$. Далее из (2.12) следует искомое выражение для порога:

$$
m_{a}=\frac{\pi}{2} \sqrt{1+a_{m}^{2}} \text { или }(h H)_{a}=\frac{\pi}{2} \mu_{\chi} \sqrt{1+a_{m}^{2}}, \quad \omega=0,
$$

где $a_{m}=a$ при $\omega=0$. При $a_{m}=0$, когда $\Phi_{m}=0$ (колебания отсутствуют), это выражение дает известный статический порог Фредерикса

$$
(h H)_{0}=\frac{\pi}{2} \mu_{\chi}=\mu_{c}, \quad \text { но } h H<\frac{\pi}{2} \mu_{\chi} \text { или } m<\frac{\pi}{2} .
$$

Здесь неравенство следует из (3.1) с учетом обозначений (2.14) и выражает условие статической устойчивости недеформированной структуры нематика $(\Phi=0)$ в магнитном поле.

В области устойчивых состояний $(m<2 / \pi)$ система характеризуется минимумом потенциальной энергии, в котором она находится при $\omega=0$. С переходом же в область неустойчивых состояний $(m>2 / \pi)$ на дне потенциальной ямы возникает (вначале невысокий) максимум, а с обеих его сторон - два минимума и два устойчивых состояния (рис. 3). Переходы между ними являются теми симметричными колебаниями, которые мы рассматриваем. Поэтому при $\omega=0$ система находится на максимуме.

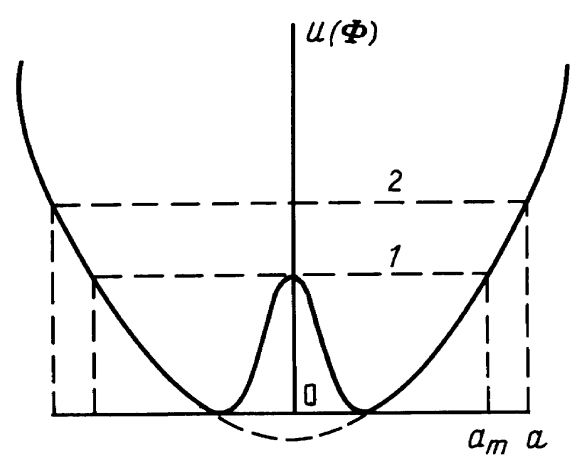

Рис. 3. Зависимость упругой энергии от угловой координаты $\Phi$. 
На рис. 3 показана минимальная амплитуда $a_{m}=\operatorname{tg} \Phi_{m} / 2$, при которой еще возможно преодоление потенциального барьера (уровня энергии 1 на рисунке), т.е. динамическое состояние с частотой $\omega \neq 0$. Амплитуде $a>a_{m}$ отвечает более высокий уровень энергии 2 . Величина амплитуды $a_{m}$ определяется в первую очередь произведением $(h H)$. Соответствуюшие соотношения возникают путем объединения (3.1) и (3.2). В результате получим

$$
h H \leqslant \frac{\pi}{2} \mu_{\chi} \sqrt{1+a_{m}^{2}} \text { или } a_{m}^{2} \geqslant \frac{4 h^{2} H^{2}}{\pi^{2} \mu_{\chi}^{2}}-1=\frac{h^{2} H^{2}}{\left(h^{2} H^{2}\right)_{0}}-1 .
$$

Знаку равенства отвечает формула, определяющая минимальную амплитуду $a_{m}$. Соответствуюшая ветвь колебаний начинается в точке $m_{a}^{-1}=\mu_{\chi} / h H$. Очевидно, что соотношения (3.4) имеют смысл, если произведение $(h H)$ превосходит статический порог $\Phi$ редерикса $(h H)_{0}$. Знак неравенства определяет лежащую справа от точки $\mu_{\chi} / h H$ область сушествования колебаний с амплитудой $a_{m}$. Ясно, что чем выше и толше слой, тем больше минимальная амплитуда колебаний $a_{m}$. Это обусловлено тем, что дестабилизирующее поле снижает упругое сопротивление слоя, способствуя возникновению колебаний: чем оно больше, тем большие угловые отклонения возможны.

Все соотношения (3.1)-(3.4) относятся, как уже отмечалось, к статическим предельным условиям при $\omega=0$. Далее же нас будет интересовать процесс затухания колебаний малых, но конечных частот $\omega$ при постоянных внешних условиях $h H=$ const или $m^{-1}=$ const. Этим условиям отвечает на рис. 2 вертикальная прямая (I) $m_{a}^{-1}=$ const, пересекающая семейство ветвей, амплитуды колебаний которых превышают амплитуду $a_{m}$, соответствующую согласно (3.4) точке $m_{a}$ на оси абсцисс. Затуханию отвечает спуск по этой прямой в сторону меньших амплитуд вплоть до предела $a_{m}$, когда не преодолевается уже потенциальный барьер между двумя состояниями равновесия $\pm \Phi$. Сушественно, что вместе с уменьшением амплитуд $\left(a \rightarrow a_{m}\right)$ происходит и уменьшение фазовой скорости $\left(C=\omega m^{-1}=h T^{-1}\right)$ вплоть до нуля $\left(C \rightarrow C\left(a_{m}\right)=0\right)$. Эта особенность дисперсии низкочастотных участков ветвей колебаний является наиболее интересным моментом, определяющим аномально низкое затухание колебаний.

\section{4. ЗАТУХАНИЕ НИЗКОЧАСТОТНЫХ МОД}

В ряде случаев затухание нелинейных крутильных колебаний может быть невелико несмотря на значительную врашательную вязкость среды $\gamma$, при которой линейные колебания сильно затухают. Некоторые соображения в пользу этого явления были изложены во введении.

Перейдем теперь к теории, которая будет развита в рамках традиционного подхода - адиабатического или слабонеравновесного приближения. При этом предполагается, что при слабом затухании колебаний интегральная энергия (всего слоя $h$, усредненная также за полупериод $T) E$ не остается постоянной, как в случае бездиссипационной среды $(\gamma=0)$. Она эволюционирует в соответствии с законом баланса энергии

$$
\begin{aligned}
\frac{d E}{d t} & =-2 D, \quad D=\frac{1}{2} \gamma \int_{0}^{T} \int_{-h / 2}^{h / 2}\left(\partial \Phi / \partial t^{\prime}\right)^{2} d x d t^{\prime} \\
E & =\frac{1}{2} \int_{0}^{T} \int_{-h / 2}^{h / 2}\left[J\left(\partial \Phi / \partial t^{\prime}\right)^{2}+k(\partial \Phi / \partial x)^{2}-\chi_{i k} H_{i} H_{k}\right] d x d t^{\prime} .
\end{aligned}
$$


Здесь $D$ - диссипативная функция, которая выбирается в квадратичной форме из-за малой величины угловой скорости $\partial \Phi / \partial t^{\prime}$, что реализуется в рассматриваемых низкочастотных модах, $t^{\prime}$ - “быстрое” время. В предыдушем разделе было показано, что эти моды возникают в дестабилизируюшем поле и характеризуются при достаточно больших (закритических) магнитных полях или больших толщинах слоя $h$ весьма низкими фазовыми скоростями $C=\omega m=h / T$.

В случае линейных колебаний, как известно, в первую очередь происходит эволюция не частоты колебаний, а их амплитуды. Первая же приобретает лишь постоянную добавку, пропорциональную коэффициенту затухания.

В нелинейном же случае не только амплитуда $a=\operatorname{tg} \frac{\Phi_{e}}{2}$, но и частота (или фазовая скорость $C=h / T)$ убывают со временем, поскольку эти величины теперь взаимосвязаны: $C=C(a)$.

Уравнение эволюции амплитуды следует из уравнения баланса энергии (4.1), где $E(a)$ и $D(a)$ являются согласно (4.2) усредненными по быстрым движениям (по $\left.t^{\prime}\right)$ величинами, полученными на основании бездиссипативных решений вида (2.7), (2.9). Далее вводится медленное (эволюционное) движение, т.е. принимается, что $a=a(t)$, $\partial a / \partial t \ll T^{-1}$. Последнему условию адиабатичности будет дано в дальнейшем оправдание. Здесь $t$ - "медленное" время. Уравнение (4.1) позволяет в принципе найти функцию $a(t)$. Подставляя ее в формулы (2.7) (2.9), получим приближенное решение уравнения затухающих колебаний (1.3) в виде

$$
\operatorname{tg} \frac{\Phi\left(x, t, t^{\prime}\right)}{2}=a(t) \operatorname{cn}\left(\frac{x}{h} 2 K_{2}\right) \operatorname{cn}\left(\frac{t^{\prime}}{h} C(a) 2 K_{1}\right), \quad a(t)=\operatorname{tg} \frac{\Phi_{e}(t)}{2}
$$

Разумеется, нет возможности строго реализовать эту программу. Неизбежны некоторые допущения.

Одно из них связано с переходом к асимптотическому виду эллиптических функций в решении (2.7). Целесообразно рассмотреть предельньй случай $\left(\nu_{1} \simeq 1, \nu_{2} \simeq 0\right)$, когда в этой формуле можно принять, что

$$
\operatorname{cn}\left(\frac{x}{h} 2 K_{2}\right) \simeq \cos \frac{\pi x}{h}, \quad \operatorname{cn}\left(\frac{t^{\prime}}{T} 2 K_{1}\right) \simeq 1 / \operatorname{ch} \frac{\pi t^{\prime}}{T}
$$

Этой асимптотике соответствуют интересуюшие нас начальные участки дисперсионных ветвей, исходящих из точек с абсциссами $m_{a}^{-1}=\frac{\pi}{2} \sqrt{1+a_{m}^{2}}$ на горизонтальной оси (см. рис. 2). Именно этим низкочастотным участкам и соответствуют слабозатухающие колебания, что прямо связано с малыми фазовыми скоростями $(C \rightarrow 0)$.

Оказывается, что затухание убывает до нуля с приближением по вертикали (I) $m_{a}^{-1}=$ const к пределам $a=a_{m}, C=0$ (рис. 2). Поэтому для интересующего нас нижнего участка вертикали имеем

$$
C \simeq C^{\prime}\left(a-a_{m}\right), \quad a_{m}=\min a \text { при } \omega \rightarrow 0 .
$$

Величина $a_{m}$ есть также пороговое значение амплитуд. Колебания с амплитудами меньшими чем $a_{m}$ при заданном поле и толщине слоя не возникают. 
Перейдем теперь к построению уравнения амплитудной эволюции. Для этого нужно вначале провести интегрирование в (4.2) по координате $x$ и быстрому времени $t^{\prime}$, стоящим в аргументах эллиптических косинусов в (4.3). При этом амплитуда $a$ условно считается константой. В результате получаются выражения

$$
E=\pi J h^{-1} C^{2}(a) w(a)+\pi k h^{-1} u(a), \quad D=\pi \gamma h^{-1} C^{2}(a) w(a) .
$$

Явные формулы для $w(a)$ и $u(a)$ сушествуют, однако мы ограничимся здесь общим анализом.

Используем теперь медленное время $t$, т.е. положим, что $a=a(t), \partial a / \partial t \ll T^{-1}$. Последнее условие является условием малости величины затухания. Тогда уравнение баланса энергии (4.1) примет вид уравнения амплитудной эволюции

$$
\frac{\partial E}{\partial a} \frac{\partial a}{\partial t}=-2 D(a)
$$

Теперь запишем это уравнение в интегральной форме, умножив предварительно обе части на $C(a) / 2 h$,

$$
-\frac{1}{4 h} \int_{a}^{a_{n}} \frac{C(a)}{D(a)} \frac{\partial E}{\partial a} d a=n(a, t), \quad n(a, t)=\frac{1}{2 h} \int_{t}^{t_{n}} C d t .
$$

Здесь вместо абсолютного времени затухания $\int d t$ введена относительная величина $n=$ $\int \frac{d t}{2 T}$, т.е. число периодов колебаний, необходимых для полного затухания амплитуды. Эта величина более определенно характеризует затухание, поскольку она учитывает эффект увеличения и самого периода колебаний $2 T$ с приближением процесса к пороговому.

В (4.8) пределы интегрирования $a$ и $t$ относятся к некоторому началу отсчета процесса затухания. Что касается пределов $a_{n}$ и $t_{n}$, то по этому поводу нужно отметить следующее. Особый интерес представляет последняя стадия процесса затухания, когда достигается значение амплитуды $a_{n}$, близкое к минимальному: $a_{n} \simeq a_{m}$. Рассматривается лишь малый участок изменения амплитуды $\left(a-a_{n}\right) \ll 1$, которому соответствует время затухания $\Delta t=\left(t_{n}-t\right)$. Это позволяет получить асимптотический закон эволюции в простой форме.

Запишем выражение (4.8) согласно (4.6), (4.7) в виде

$$
n=n_{1}+n_{2},
$$

здесь

$$
n_{1}=-\frac{\pi J}{4 \gamma h^{2}} \int_{a}^{a_{n}} \frac{\partial\left(C^{2} w\right) / \partial a}{C^{2} w} d a, \quad n_{2}=-\frac{\pi k}{4 \gamma h^{2}} \int_{a}^{a_{n}} \frac{u^{\prime}}{C} d a, \quad u^{\prime}=\frac{1}{w} \frac{\partial u}{\partial a} .
$$

Переход от абсолютного времени затухания $\Delta t$ к относительному $n$ вызван появлением бесконечного времени процесса колебаний $\Delta t$ вблизи порога $a_{n}=a_{m}$. Интегралы же в (4.10) обладают лучшей сходимостью без потери смысла характеристики затухания. 
Запишем первый из них вначале так:

$$
\int_{1}=2 \int_{C}^{C_{n}} d C+\int_{a}^{a_{n}} C \frac{\partial \ln w}{\partial a} d a .
$$

Во втором слагаемом стоят два подынтегральных множителя. Первый $(C)$ имеет согласно (4.5) порядок $\left(a-a_{n}\right)$. Что касается второго, то даже если в его ряду Тейлора по степеням разности $\left(a-a_{n}\right)$ удержать член нулевой степени, то второе слагаемое в (4.11) все равно даст члены более высокого порядка малости, чем первое. Выписывая его в явной форме, получим

$$
n_{1} \simeq \frac{\pi J}{2 \gamma h^{2}}\left(C-C_{n}\right)+O\left[\left(a-a_{n}\right)^{2}\right] .
$$

Очевидно, что величина $n_{1}$ остается регулярной при $a_{n} \rightarrow a_{m} \neq 0, C_{n} \rightarrow C_{m}$.

Второй интеграл в (4.10) после элементарных преобразований с учетом (4.5) (а также разложения $u^{\prime}(a)$ по степеням $\left.\left(a-a_{n}\right)\right)$ примет вид

$$
\begin{aligned}
\int_{2} & =\frac{u^{\prime}\left(a_{n}\right)}{C^{\prime}} \int_{C}^{C_{n}} \frac{d C}{C}+\frac{\partial u^{\prime}}{C^{\prime} \partial a_{n}} \int_{C}^{C_{n}} d C+O\left[\left(a-a_{n}\right)^{2}\right] \simeq \\
& \simeq-\frac{u^{\prime}\left(a_{n}\right)}{C^{\prime}} \ln \frac{C}{C_{n}}-\frac{\left(C-C_{n}\right)}{C^{\prime}} \frac{\partial u^{\prime}}{\partial a_{n}} .
\end{aligned}
$$

Используя (4.13) в (4.10), имеем

$$
n_{2}=\frac{\pi k u^{\prime}\left(a_{n}\right)}{2 \gamma h^{2} C^{\prime}} \ln \frac{C}{C_{n}}+\frac{\pi k\left(C-C_{n}\right)}{2 \gamma h^{2} C^{\prime}} \frac{\partial u^{\prime}}{\partial a_{n}}, \quad C_{n} \gtrsim C_{m}=0,
$$

здесь $C$ рассматривается как функция вида (4.5). Тогда обрашаясь к (4.9), получим в окрестности порога неустойчивости структуры нематика следуюший асимптотический закон затухания колебаний в малом амплитудном интервале:

$$
n(a)=\frac{\pi}{2 \gamma h^{2}}\left[J+\frac{k}{C^{\prime}} \frac{\partial u^{\prime}}{\partial a_{n}}\right]\left[C(a)-C_{n}\right]+\frac{\pi k u^{\prime}\left(a_{n}\right)}{4 \gamma h^{2} C^{\prime}} \ln \frac{C(a)}{C_{n}} .
$$

Если нижний предел этого интервала $a_{n}$ достигает порога $a_{m}$, то $C_{n}=C_{m}=0$ и второе слагаемое (4.15) расходится. Это значит, что время затухания становится бесконечно больше периода колебаний, т.е. затухание на границе сушествования колебаний исчезает. Исключая этот крайний случай, будем считать, что $a_{n}>a_{m}$. Тогда $\left(C-C_{n}\right) \gtrsim C_{n} \simeq 0$ и логарифмический член хотя и велик, но ограничен. Опуская линейньй член в (4.15), получим закон эволюции в виде

$$
n(a) \simeq \frac{\pi}{4} \frac{k u^{\prime}\left(a_{n}\right)}{\gamma h^{2} C^{\prime}} \ln \frac{C(a)}{C_{n}} .
$$

В этом приближении из закона исчезает инерционньй член $(\sim J)$, т.е. эволюция приобретает диффузионный характер. Используя законы эволюции (4.15), (4.16), можно 
после установления зависимости $a(t)$ в явном виде получить с помощью (4.3) решение уравнения затухаюших колебаний (1.3), разумеется, в указанных выше пределах.

Выявленные особенности затухания ориентационных колебаний прямо связаны с дестабилизирующим воздействием магнитного поля. Если же поле направлено не ортогонально, а параллельно граничной ориентации или однородной ориентации всего слоя в недеформированном состоянии, то результаты получаются совершенно иными. В этом случае в уравнениях (1.3), (1.4) изменяется на противоположный знак перед нелинейным членом, колебания происходят в потенциальной яме (с одним минимум) около устойчивого состояния равновесия. Кроме того, дисперсионные ветви колебаний зеркально симметричны (относительно асимптоты $C=C_{0}$ ) ветвям, показанным на рис. 2. Они не имеют низкочастотных участков, и каждая простирается вьше некоторой граничной частоты. С ними связаны и большие значения фазовых скоростей $C \geqslant C_{0}$. Аномально низкое поглошение таких колебаний отсутствует.

Работа выполнена при финансовой поддержке Российского фонда фундаментальных исследований (проект № 96-01-01150).

\section{Список литературы}

[1] П. де Жен. Физика жидких кристаллов. М.: Мир, 1977.

[2] Э. Л. Аәро. Автореф. докт. дис. физ.-мат.н. Л.: ЛГУ, 1982.

[3] Э. Л. Аәро, А.Н. Бульъъин. Прикл. матем. и мех. 1971. Т. 35. № 5. С. 879.

[4] Д. Л. Ләм. Введение в теорию солитонов. М.: Мир, 1983. С. 153.

[5] Э. Л. Аәро. Кристаллография. 1995. Т. 40. В. 5. С. 889.

[6] Солитоны в действии. Ред. К. Лонгрен, Э. Скотт. М.: Мир, 1981. Гл. 8.

[7] E. Янке, Ф. Эмде, Ф. Лёи. Специальные функции. М.: Наука, 1977. Гл. 9.

Поступила в редакцию 21.V.1996 г.

\section{E. L. Aero \\ WEAK DAMPING SLOW MODES OF ORIENTED NONLINEAR OSCILLATIONS IN LIQUID CRYSTALLS IN UNSTABILIZING MAGNETIC FIELDS}

Nonlinear inertial torsion oscillations of the structure of nematic liquid crystall in unstabilizing magnetic field have low frequency modes possessing the anomaly weak damping. The corresponding solution of the sine-Gordone type equation to the account for viscous rotating friction describes the transitions via the potential barrier between two equillibrium states. We show that when the oscillation amplitude is large enough to overcome the barrier, the damping time for the period of oscillation diverges logarithmically. 